\title{
Co-Increasing Neuronal Noise and Beta Power in the Developing Brain
}

Wei He $\mathrm{H}^{1,2}$, Thomas Donoghue ${ }^{3}$, Paul F Sowman ${ }^{1,2}$, Robert A Seymour ${ }^{1,2}$, Jon Brock ${ }^{1,2}$,

1 Department of Cognitive Science, Macquarie University, 16 University Avenue, Sydney, 2109, NSW, Australia

2 Australian Research Council Centre of Excellence in Cognition and Its Disorders, 16 University 7 Avenue, Sydney, 2109, NSW, Australia

3 Department of Cognitive Science, University of California, 9500 Gilman Dr, La Jolla, CA 92093,

4 Department of Linguistics, Macquarie University, 16 University Avenue, Sydney, 2109, NSW, Australia

14 Corresponding Author: Wei He, +61 (02) 9850 2952; wei.he@mq.edu.au 


\section{ABSTRACT}

16 Accumulating evidence across species indicates that brain oscillations are superimposed 17 upon an aperiodic $1 / f$ - like power spectrum. Maturational changes in neuronal oscillations 18 have not been assessed in tandem with this underlying aperiodic spectrum. The current study 19 uncovers co-maturation of the aperiodic component alongside the periodic components 20 (oscillations) in spontaneous magnetoencephalography (MEG) data. Beamformerreconstructed MEG time-series allowed a direct comparison of power in the source domain between 24 children ( $8.0 \pm 2.5$ years, 17 males $)$ and 24 adults ( $40.6 \pm 17.4$ years, 16 males $)$.

23 Our results suggest that the redistribution of oscillatory power from lower to higher frequencies

24 that is observed in childhood does not hold once the age-related changes in the aperiodic signal are controlled for. When estimating both the periodic and aperiodic components, we found that power increases with age in the beta band only, and that the $1 / f$ signal is flattened in adults compared to children. These results suggest a pattern of co-maturing beta oscillatory power with the aperiodic $1 / f$ signal in typical childhood development. 


\section{INTRODUCTION}

30

\section{Neuronal Oscillations Characteristic of Childhood Brain Maturation}

Neuronal oscillatory power undergoes profound developmental changes throughout childhood (Gomez et al., 2017; Rodriguez-Martinez et al., 2017). These developmental changes in neuronal oscillations are often assessed noninvasively using electrophysiological brain recordings such as magneto-/electro-encephalography (MEG/EEG). Commonly, a Fourier analysis is used to compute the power spectral density (PSD) in fixed frequency bands, including delta $(1-4 \mathrm{~Hz})$, theta $(4-8 \mathrm{~Hz})$, alpha $(8-13 \mathrm{~Hz})$, beta $(13-30 \mathrm{~Hz})$, and gamma $(30-80$ $\mathrm{Hz}$; Mackay, 1997; note that different studies may differ slightly in establishing the boundaries between frequency bands). There are age-related decreases in total power, which is estimated across a broad frequency range (Gasser et al., 1988; Schafer et al., 2014; Gomez et al., 2017; Rodriguez-Martinez et al., 2017), as well as age-related decreases in absolute power in each of the narrowband frequencies (Gasser et al., 1988; Boord et al., 2007). Studies also report a low-to-high redistribution of relative power (i.e., where power is estimated in any given band in relation to the total power across all frequencies); more specifically, relative power decreases in the delta and theta bands and increases in the alpha, beta and gamma bands (Puligheddu et al., 2005; Gomez et al., 2013; Schafer et al., 2014). In addition, there is an increase during childhood in the peak frequency of alpha oscillations, which typically reach a peak frequency of $\sim 10 \mathrm{~Hz}$ around the primary/elementary school years (Marcuse et al., 2008; Boersma et al., 2011; Cragg et al., 2011; Smit, Boomsma, et al., 2012; Miskovic et al., 2015; Gomez et al., 2017; Rodriguez-Martinez et al., 2017).

Power in each narrow frequency band has been associated with different cognitive functions. The alpha rhythm (Markand, 1990) has been prominently associated with inhibition of visual attention (Jensen and Mazaheri, 2010; Clayton et al., 2017; Voytek et al., 2017). In addition, the increase of alpha peak frequency with age has been considered a biomarker for cognitive 
development (Marcuse et al., 2008; Boersma et al., 2011; Cragg et al., 2011; Smit, Boomsma, et al., 2012; Miskovic et al., 2015; Gomez et al., 2017; Rodriguez-Martinez et al., 2017), which suggests that the perception of visual stimuli would also improve with age (Thut et al., 2012). In the sensory motor cortex, mu, which is analogous to the alpha band (Mackay, 1997), and beta oscillations have been found to increase when cortical motor areas are disengaged (Ritter et al., 2009; Jenkinson and Brown, 2011). Our recent longitudinal MEG study of motor development in children demonstrated linear increases in amplitude and mean frequency in movement-evoked mu and beta oscillations (Johnson et al., 2019). In studies of developmental resting-state neuronal activity (i.e., in the absence of any specific cognitive event), there are also reports of age-related increases in mu power from infancy to age 5 (Berchicci et al., 2011) and age-related increases in beta power between age 9-14 and 20-42 (Heinrichs-Graham et al., 2018). Delta power has been correlated with different stages of sleep (Amzica and Steriade, 1998), and theta activity has been shown to relate to executive attention and working memory (Wang et al., 2005). Developmental trends in delta and theta power are less clear, with some studies reporting profound decreases during childhood (Schafer et al., 2014; Gomez et al., 2017) and others reporting no changes between 9 and 11 years, followed by decreases into early adulthood (Campbell and Feinberg, 2009). There is evidence of increased gamma activity in the initiation and cessation of movement (Gaetz et al., 2010; Burianova et al., 2013; Cheyne and Ferrari, 2013; Marstaller et al., 2014; Sowman et al., 2014). Gamma activity can be adult-like as early as 3 years of age in motor tasks (Johnson et al., 2019), and resting state gamma activity across the first 3 years of life is predictive of later development of language and cognitive skills (Benasich et al., 2008). Based on the previous literature, it is clear that a precise characterisation of developmental changes in neuronal oscillatory power is critical for our mechanistic understanding of the maturation of cognitive functions during childhood. 


\section{Outstanding Questions}

80

Spectral analysis of resting-state MEG/EEG recordings has proven to be a powerful tool for assessing age-related power changes. However, nearly all previous resting-state studies have used the five canonical frequency bands to estimate developmental changes in power. These studies, therefore, are susceptible to methodological challenges. For example, examining power in pre-defined bands can conflate power changes with other parameters, such as oscillation centre frequency and bandwidth (Haller et al., 2018). An example is the increase in alpha peak frequency with age, which is considered to be one of the most important electrophysiological hallmarks of brain development (Valdés et al., 1990). Following historical tradition, nearly every study to date has defined the peak frequency as the frequency with the highest amplitude within the range of the canonical alpha band. Under this constraint, it is impossible to determine whether or not changes in the peak frequency may in fact reflect shifts of the peak frequency outside the canonical alpha band. Similarly, group-level estimates of power in one band may leak into the estimates of power in adjacent bands, given the variability in oscillation centre frequency across individuals (Haegens et al., 2014; Samaha and Postle, 2015) and age (Rodriguez-Martinez et al., 2017). But perhaps most importantly, the narrowband estimates of oscillatory activity may be affected by aperiodic components in the signal. Brain activity at many spatiotemporal scales, ranging from neuronal membrane potentials to MEG/EEG signals, exhibits an aperiodic background signal that co-exists with neuronal oscillations (He, 2014). This aperiodic signal follows a power-law function: $P \propto 1 / f^{x}$. Thus, power $P$ is inversely proportional to frequency $f$ with a power-law exponent of $x$, which is equivalent to the slope of the power spectrum when plotted in the log-log space. The aperiodic $1 / f$ signal is not only prevalent in the nervous system, but it is a ubiquitous feature of a wide variety of time-varying real-world systems, including the flow of the river Nile and the luminosity of stars (Bak et al., 1987). Historically, the aperiodic $1 / f$ signal has not been as well investigated as periodic (oscillatory) brain activity, resulting in a lack of consensus on how 
to measure it, what it may reflect, and what might be its physiological generators. Nonetheless, the recent electrophysiological literature has started to elucidate the functional significance of the $1 / f$ signal in human cognition and behaviour. For instance, this signal has been found to vary systematically with age (Voytek et al., 2015), and to change with task demands (He et al., 2010) that can co-vary with behavioural performance (Podvalny et al., 2015). Moreover, recent simulations, as well as empirical data from rats and macaques, indicate that neuronal excitation and inhibition in cortical circuits can be inferred from the slope of the invasivelyrecorded electrophysiological power spectrum (Gao et al., 2017). This further emphasises the importance of quantifying the $1 / f$ signal and its contributions to human cognition (Voytek and

114 Knight, 2015).

115 Related to this, standard analytic approaches, which estimate power in narrow frequency bands, fail to examine whether an oscillation - a rhythmic component that peaks at a particular frequency - is truly present in the power spectrum. The power that is assessed within a predefined frequency range of the electrophysiological power spectrum is most likely a mixture of both oscillatory and aperiodic $1 / f$ components. It is imperative to disentangle age-related changes in the narrowband oscillations from those in the broadband aperiodic $1 / f$ signal. Although these signals are inter-related, they are likely to represent distinct underlying neural mechanisms (Haller et al., 2018).

\section{Current Study: Aims and Hypotheses}

124 To overcome the limitations of previous studies that used narrowband power analyses, the present study used advanced analysis techniques (Haller et al., 2018) to investigate, and to disentangle, age-related effects in the aperiodic $1 / f$ and the oscillatory components of brain activity. For this, we used a paediatric whole-head MEG scanner (Johnson et al., 2010) to collect resting-state electrophysiological signals from children ranging in age from 4 to 12 years, as well as a conventional MEG scanner to obtain the same signals from adults. Importantly, source waveforms were computed using an atlas-based beamforming approach 
131 for power spectra analyses (Hillebrand et al., 2012; Hillebrand et al., 2016), which allowed for

132 direct comparison of MEG data acquired from the two systems (He et al., 2019).

133 We first analysed neuronal power in pre-defined frequency bands using standard methods, to 134 permit direct comparisons of our results with those from previous studies. In line with the previous literature (Gasser et al., 1988; Puligheddu et al., 2005; Marcuse et al., 2008; Boersma et al., 2011; Cragg et al., 2011; Smit, Boersma, et al., 2012; Gomez et al., 2013; Schafer et al., 2014; Miskovic et al., 2015; Gomez et al., 2017; Rodriguez-Martinez et al., 2017), we hypothesised that, in contrast to children, adults would show decreased low-frequency power, increased high-frequency power, and an increased alpha peak frequency.

140 Subsequently, we used an automatic parameterising algorithm (Haller et al., 2018) that efficiently disentangles the two features - the slope and the offset - that characterise the $1 / f$ signal, and the three features - the centre frequency, power and bandwidth - that characterise

143 the oscillatory components. Since the automatic parameterising algorithm does not impose 144 band boundaries, this method allows for the assessment of group and individual differences 145 in the centre frequency, power and bandwidth of the oscillations, both in the broadband spectra and in pre-defined narrow bands. With regard to possible age-associated differences in $1 / f$ signal, there exists only one developmental fMRI/EEG study so far, which showed that the $1 / f$ slope was significantly flatter in 17 healthy adults compared to 21 full-term newborns

149 (Fransson et al., 2013). Based on the limited developmental MEG/EEG evidence regarding 150 the $1 / f$ signal, we hypothesised that differences in the $1 / f$ signal may account to a large 151 extent for observed age-related power differences between children and adults, and that 152 canonical frequency band analyses may confound some band specific power changes with $1531 / f$ signal shifts. In particular, we predicted that the $1 / f$ slope would be flatter, and the offset 154 would be smaller in adults as compared to children (Fransson et al., 2013). 


\section{Participants and Ethics Statement}

157 This study included 52 human participants ( 28 children and 24 adults), namely healthy controls

158 that had been recruited in a larg project on stuttering. Data from 4 children were excluded from

159 the analysis due to excessive head movement (> $5 \mathrm{~mm}$ ), incidental system noise or signs of 160 drowsiness throughout the recording. Drowsiness was monitored online through a video161 camera so that any affected data would be removed from further analysis. Child participants were accompanied by an experienced researcher who sat with them during the whole session to make sure they remained comfortable, and who monitored and encouraged their compliance. The final sample consisted of 24 children $(8.0 \pm 2.5$ years, 17 males $)$ and 24 adults (40.6 \pm 17.4 years, 16 males).

Written informed consent was obtained from the adult participants and from the parents/guardians of the children prior to the experiment. No participant reported personal or family history of neurological disease or psychological impairment and none were taking medication that could affect MEG recordings at the time of participation. All participants were remunerated \$AUD 40 for their participation. The experimental procedures were approved by the Human Participants Ethics Committee at Macquarie University.

\section{MEG Data Acquisition}

173 Resting-state MEG data of 300 seconds were acquired for child and adult participants using

174 two separate whole-head gradiometer MEG systems. Child data were acquired using a 175 paediatric 125-channel whole-head gradiometer MEG system (Model PQ1064R-N2m, 176 Kanazawa Institute of Technology/KIT, Kanazawa, Japan). Adult data were acquired using a 177 160-channel whole-head gradiometer MEG system (Model PQ1160RN2, KIT, Kanazawa, 178 Japan). Use of the paediatric MEG system to overcome critical limiting factors for MEG 179 experimentation on children below the ages of five to six years (Irimia et al., 2014), including 
180 a much smaller head size and overall structure in children (the smaller crown to shoulder 181 distance prevents the full insertion of the head into the adult helmet), has been demonstrated 182 previously (Sowman et al., 2014; He, Brock, et al., 2015; He, Garrido, et al., 2015; Etchell et 183 al., 2016).

184 The gradiometers of both systems have $50 \mathrm{~mm}$ baseline and $15.5 \mathrm{~mm}$ diameter coils positioned in a glass fibre reinforced plastic cryostat for measurement of the normal component of the magnetic field from the human brain (Kado et al., 1999). In both systems, neighbouring channels are $38 \mathrm{~mm}$ apart and $20 \mathrm{~mm}$ from the outer dewar surface. These factors ensure that the signals obtained by the two MEG systems are equivalent. The 125channel dewar was designed to fit a maximum head circumference of $53.4 \mathrm{~cm}$, accommodating more than $90 \%$ of heads of 5 -year-olds (see Johnson et al., 2010 for details). Both systems were situated within the same magnetically shielded room within the KITMacquarie Brain Research Laboratory (https://www.mq.edu.au/research/research-centresgroups-and-facilities/healthy-people/facilities/meg), and therefore environmental noise was comparable.

During MEG data acquisition, participants were asked to remain relaxed, awake and with their eyes fixed on a white cross at the centre of a black $36 \mathrm{~cm}$ (width) $\times 24 \mathrm{~cm}$ (length) rectangular image with $4 \times 4$ degrees of visual angle. Visual display was presented on a back-projected screen mounted approximately $140 \mathrm{~cm}$ above the participant using video projectors situated outside the magnetically shielded room (child MEG projector: Sharp Notevision Model PG10S,

200 Osaka, Japan; Adult MEG projector: InFocus Model IN5108, Portland, USA). An overview of 201 the child-friendly experimental protocol can be found in the video article (Rapaport et al., 2019).

\section{MEG Data Processing}

203 An overview of the processing pipeline is illustrated in Figure 1. MEG data were acquired at a sampling frequency of $1000 \mathrm{~Hz}$, using a hardware bandpass filter of $0.03-200 \mathrm{~Hz}$. The 
continuous raw MEG data were filtered off-line from 0.5 to $100 \mathrm{~Hz}$ using bi-directional IIR Butterworth filters with DC removal and segmented into epochs of 4096 samples $(=4.096$ seconds). The data were visually inspected by $\mathrm{WH}$, and epochs that contained oculographic, myographic, and system/environmental artefacts (e.g., squid jumps) were removed. The first and last epochs were also excluded from the analysis. A mean of $23.8 \pm 3.02$ artefact-free epochs of $4.096 \mathrm{~s}$ data in children (15-28 epochs) and $40.0 \pm 0.02$ artefact-free epochs in adults (39-40 epochs) were selected for subsequent source modelling. There were agerelated differences in the number of clean trials between children and adults $(t(46)=26.31, p$ $<0.01$; two-sample t-test using the ttest2 function in MATLAB, version R2017b), as expected and was inevitable due to the fact that more trials were removed from younger participants because of movement. However, it has been shown in previous simulations that beamformer performance plateaus before the lower limit of $\sim 80$ seconds of data that was used for our analysis (20 epochs of 4.096 seconds; Brookes et al., 2008).

MEG sensor data were then projected onto a parcellated cortical surface using an atlas-based beamforming approach (Hillebrand et al., 2012; Hillebrand et al., 2016), providing source activities at each centroid of the automated anatomical labelling (AAL) atlas (Tzourio-Mazoyer et al., 2002). Firstly, the geometry of each participant's scalp was reconstructed from a "surrogate" MRI, where the Montreal Neurological Institute (MNI) template T1 structural brain image was warped to each participant's digitized head shape with an iterative closest point algorithm implemented in BrainWave (Cheyne et al., 2014). Secondly, a multi-sphere volume conductor model was calculated using the outline of the scalp from this co-registered data in MRIViewer of the CTF MEG5 software (VSM MedTech Systems Inc., Coquitlam BC, Canada; Version 5.0.2). Thirdly, the broadband $(0.5-48 \mathrm{~Hz})$ data covariance matrix was calculated from all selected epochs, and a unity noise covariance was used. Lastly, the data covariance, the unity noise covariance, together with an equivalent current dipole source model and the multisphere volume conductor model, were combined to reconstruct beamformer weights for the 
232 Subsequently, the broadband MEG sensor data were projected through the normalised 233 beamformer weights in order to obtain traces of neuronal activity in the cortical space (Cheyne 234 et al., 2007).

235 To counteract trial imbalance between groups, we chose for each individual the first 15 236 artefact-free epochs from each of the $80 \mathrm{AAL}$ source regions of interest (80 Regions of 237 Interest/ROIs; 78 cortical and bilateral hippocampal) for the subsequent estimation of power 238 spectral density. 


\section{A. Resting State MEG Recording}

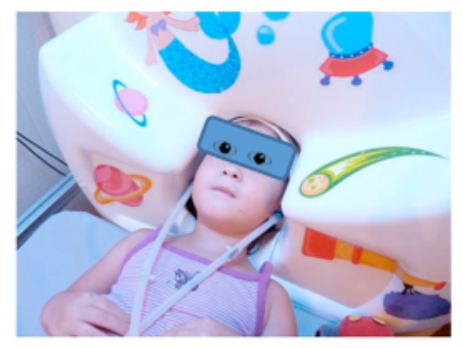

D. Head Shape and Template MRI warping
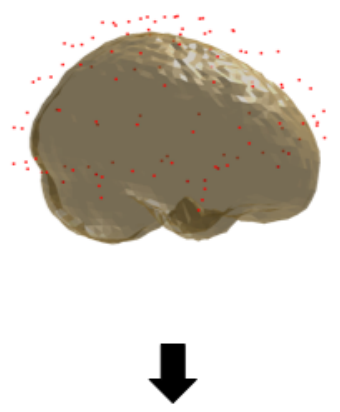

E. Beamforming onto Parcellated Brain

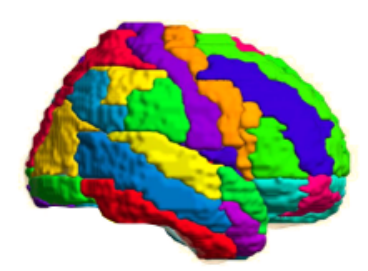

B. Bandpass Filtered Data

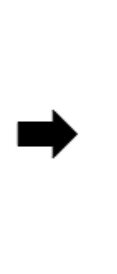

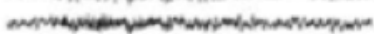

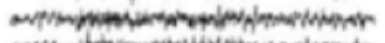

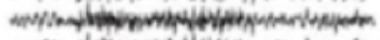

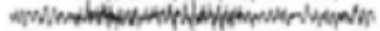

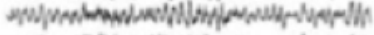

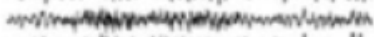

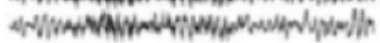

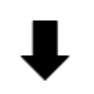

C. MEG and Head Shape Co-registration

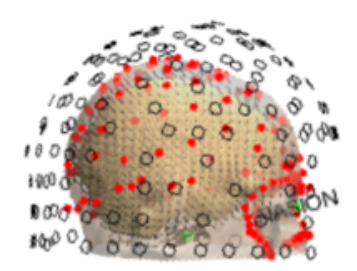

F. Source Data per Brain Area

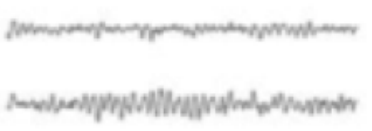

240 Figure 1. Schematic overview of the processing pipeline. The eyes-open resting state MEG were recorded (A) and bandpass filtered (B) before being coregistered to individual head shape (C) and template MRI (D). Following this,

243 data were epoched and beamformed to parcels of the AAL atlas (E). Power 244 spectral density was then estimated from individual cortical sources by Welch's 245 method (F). 


\section{Spectral Analysis}

\section{Conventional Analysis in a priori Defined Frequency Bands}

248 Power spectral density (PSD) was estimated for each participant, ROI and artefact-free 249 epochs separately using Welch's method (Welch, 1967) implemented in MATLAB 2017b, with $25050 \%$ overlap and a Hamming window of 3 s (resulting in a spectral resolution of $0.24 \mathrm{~Hz}$ ). A 251 single PSD for each participant was obtained by averaging the PSDs across all epochs and 252 ROls.

253 A conventional spectral analysis was carried out by calculating the absolute power from the 254 raw power spectrum in five canonical frequency bands (delta: $1-4 \mathrm{~Hz}$, theta: $4-8 \mathrm{~Hz}$, alpha: 255 8-13 Hz, beta: 13-30 Hz, and low gamma: 30-48 Hz). The frequency at which each participant 256 reached the peak amplitude was calculated within the $5-13 \mathrm{~Hz}$ band for children and the 8-13 $257 \mathrm{~Hz}$ band for adults using an automated local maxima algorithm (MATLAB function findpeaks). 258 The lower frequency boundary was used for children in order to account for reduced alpha 259 peak frequencies in young children when compared to adults (Klimesch, 1999; Bathelt et al., 260 2013; Mierau et al., 2016).

\section{Parameterising the Power Spectrum with no a priori Defined Frequency Bands}

The PSDs, calculated by Welch's method, were also submitted to the FOOOF v0.1.3 parameterisation model - an open source Python package (https://github.com/fooof-

264 tools/fooof/; in Python v3.7.0) - for automatic separation of periodic and aperiodic components 265 of neural power spectra (Haller et al., 2018). Briefly, the model considers the PSDs as a linear sum of aperiodic "background" neural signal ( $1 / f$ signal) and oscillations, or peaks represented by Gaussian functions in the PSD, above the $1 / f$ signal level. 


$$
P=L+\sum_{n=0}^{N} G_{n}
$$

270 with $L$ the aperiodic "background" signal and $G$ the oscillations, modelled as $N$ Gaussians.

271 The aperiodic signal is fitted, after which the aperiodic fit is subtracted from the power 272 spectrum, creating a flattened (or aperiodic-adjusted) spectrum, wherein peaks were 273 iteratively fitted by Gaussians modelled as:

$$
G_{n}=a * \exp \left(\frac{-(F-c)^{2}}{2 w^{2}}\right)
$$

where $a$ is the amplitude, $c$ is the centre frequency, $w$ is the bandwidth of the Gaussian $G$, and $F$ is the vector of input frequencies.

277 Subsequently, a peak-removed power spectrum is calculated by subtracting all fitted 278 Gaussians from the original power spectrum. Finally, an aperiodic signal is re-estimated from 279 this peak-removed power spectrum, representing the cortical $1 / f$ background signal. Both the 280 initial and final fit of the aperiodic component are fit as:

$$
L=b-\log \left(k+F^{x}\right)
$$

where $b$ is the broadband offset, $x$ is the slope, and $k$ is the "knee" parameter, which indicates where the "bend" occurs in the $1 / f$ component. In the current case of non-invasive MEG recordings, no knee was expected across the frequency range studied (Miller et al., 2009).

The FOOOF model was fitted across the frequency range of 1 to $48 \mathrm{~Hz}$ in fixed (no spectral knee) mode (peak_width_limits $=[0.5,12]$, max_n_peaks $=10$, min_peak_amplitude $=0$, and peak_threshold $=2$, aperiodic_mode $=$ 'fixed'). Goodness-of-fit of the FOOOF model is returned in terms of the $R^{2}$ of the fit.

Two parameters - the Slope $\boldsymbol{x}$ and the Offset $\boldsymbol{b}$ - defining the aperiodic $1 / f$ background signal, and three parameters - the Centre Frequency $c$, Power $\boldsymbol{a}$ and Bandwidth $\boldsymbol{w}-$ 
characterising the oscillations were returned by the FOOOF parameterisation, and entered

292 permutation statistical comparisons between the two age groups.

293 In addition, the final aperiodic signal returned from the FOOOF model was subtracted from 294 the raw power spectrum, resulting in a flattened spectrum from which the absolute alpha and beta band power was estimated again using the conventional spectral approach.

\section{Statistical Analysis}

297 Statistical analyses were performed using permutation testing as implemented in the 298 Resampling $\quad$ Statistical $\quad$ Toolkit for $\quad$ MATLAB $\quad$ 2017b 299 (https://au.mathworks.com/matlabcentral/fileexchange/27960-resampling-statistical-toolkit).

300 We used 50,000 permutations of group membership to empirically approximate the distribution 301 for the null hypothesis (i.e., no difference between groups) for each contrast. For each 302 permutation, $\mathrm{t}$-values were derived for a contrast of interest, and any $\mathrm{t}$-values for the original contrast that exceeded the significance threshold of 0.05 for the t-distribution were deemed reliable.

In addition, Bayes Factors (alternative BF/null BF; Wagenmakers, 2007; Dienes, 2011) were estimated in JASP v0.9.2 ( Quintana and Williams, 2018; https://jasp-stats.org/) to further quantify the effect size, and to facilitate the interpretation of evidence for or against the null hypothesis when comparing to the alternative hypothesis. For the alternative hypotheses of measures being larger in adults than children and vice versa, a "unit information prior" was 310 assumed with a default Cauchy prior with a scale parameter of 0.707 (Jeffreys, 1998).

311 Bayesian correlation analyses, which allows inferences on the absence of a correlation 312 between variables to be made, were also conducted in JASP. For testing the correlations, a 313 prespecified alternative hypothesis with a flat beta prior width of 1 centred around $r=0$ was 314 used for a null hypothesis $(r=0)$. An illustration of the effects of assigning a range of different 315 prior distributions (i.e., a Bayes factor robustness check) was conducted for all Bayesian tests. 
316 BFs were thresholded $>3,>10$, and $>100$ as substantial, strong, and very strong/decisive 317 evidence in favour of the alternative hypothesis, and $B F<1 / 3$ and $<1 / 10$ for substantial and 318 strong evidence for the null hypothesis (Raftery, 1995; Jeffreys, 1998). BFs that fell in-between $3191 / 3$ and 3 were taken as insufficient evidence for either hypothesis (Jeffreys, 1998; Dienes, 320 2014). 


\section{RESULTS}

\section{Raw Power Spectra With a priori Defined Frequency Bands}

\section{Decreases in Low- and Increases in High-Frequency Power}

324

325

Figure 2A\&B depicts the results of conventional spectral analyses, absolute power was computed in five a priori defined frequency bands (delta: 1-4 Hz, theta: 4-8 Hz, alpha: 8-13 $\mathrm{Hz}$, beta: $13-30 \mathrm{~Hz}$, and lower gamma: $30-48 \mathrm{~Hz}$ ). The PSD in Figure 2A shows a tendency of the grand average power for adults to be lower across lower frequency bands, and higher in higher bands, compared to children. Permutation statistics identified significantly lower power in the delta (Figure $2 \mathrm{~B}, t(46)=-6, \quad p<0.01)$ and theta $(t(46)=-3.78, \quad p<0.01$ ) bands, and higher power in the beta $(t(46)=2.74, \quad p<0.01)$ and gamma bands $(t(46)=-2.46, \quad p=$ 0.01 ) in adults compared to children. The right-corner panel in Figure2A shows that the peak frequency was significantly higher in adults $(9.99 \pm 1.30 \mathrm{~Hz})$ than in children $(7.58 \pm 1.71 \mathrm{~Hz}$, $t(46)=5.5, \quad p<0.01)$

In addition, the data were examined by estimating a Bayes factor (alternative BF/null BF), which indicates the fit of the data under the alternative hypothesis. Estimated BFs indicated decisive evidence for lower PSDs in the delta (BF > 100, 95\% Confidence Interval $=[-2.30$, 0.93]) and theta $(\mathrm{BF}=119.80,95 \% \mathrm{Cl}=[-1.58,-0.41)$ bands, higher PSDs in the beta $(\mathrm{BF}=$ $10.66,95 \% \mathrm{Cl}=[0.16,1.26])$ and gamma $(\mathrm{BF}=6.13,95 \% \mathrm{Cl}=[0.10,1.18])$ bands, and higher peak frequency $(\mathrm{BF}>10095 \% \mathrm{Cl}=[0.86,2.13])$ in adults than children. Bayes Factors suggested that there was strong evidence for the absence of a group difference in alpha band power $(\mathrm{BF}=0.16,95 \% \mathrm{Cl}=[0.01,0.44])$. 


\section{Parameterised Power Spectra Without a priori Defined Frequency Bands}

\section{Increase in $1 / f$ Slope and Decrease in $1 / f$ Offset}

344 Figure $3 \mathrm{~A}$ shows the aperiodic component of the grand average parameterised spectrum (i.e.,

345 peaks were removed from the power spectrum) for each age group. The aperiodic power

346 spectrum was flatter in adults than in children. Permutation testing confirmed that the

$3471 / f$ slope (Figure 3B) was significantly different (flatter) in adults compared to children (adults

$348=-0.89 \pm-0.12$; children $=-1.15 \pm-0.09, t(46)=8.59, p<0.01)$, and the $1 / f$ offset was smaller

349 (adults $=6.78 \pm 0.15 ;$ children $=7.11 \pm 0.17, t(46)=-6.99, \quad p<0.01$ ). Goodness-of-fit, as

350 indexed by $R^{2}$ of the modelling fit was $0.99 \pm 0.01$ for adults, and $0.99 \pm 0.01$ for children $(t(46)$

$351=-1.05, p>0.05)$, suggesting that a difference in the model fit was not the cause of observed

352 differences in the $1 / f$ signal between groups.

353 Bayes Factors indicated decisive evidence for flatter/more positive $1 / f$ slope (BF > 100, $95 \%$ $354 \mathrm{Cl}=[1.66,3.15])$ and lower $1 / f$ offset $(\mathrm{BF}>100,95 \% \mathrm{Cl}=[-2.62,-1.20])$ in adults compared 355 to children.

\section{Correlation between Age and the Aperiodic 1/f Component}

357 Bayes Factors revealed a decisive negative correlation between age and $1 / f$ offset $(r=-0.71$, $358 \mathrm{BF}>100,95 \% \mathrm{Cl}=[-0.85,-0.39])$, and a positive correlation between age and $1 / f$ slope $(r=$ $3590.62, \mathrm{BF}=35.88,95 \% \mathrm{Cl}=[0.26,0.8])$ in children. This trend became anecdotal in adults 360 (offset: $r=-0.4, \mathrm{BF}=1.56,95 \% \mathrm{Cl}=[-0.67,0.01]$; slope: $r=-0.5, \mathrm{BF}=3.7,95 \% \mathrm{Cl}=[0.14$, $3610.75])$

Power and Bandwidth Increase for Beta Power Peaks

Figure 4 shows the group comparisons for the periodic components - Centre Frequency,

364 Power, and Bandwidth - of the peak oscillation (i.e., the highest power peak across all 
frequencies in FOOOF model). Figure 4A demonstrates that $95.83 \%$ of children and $58.33 \%$ of adults exhibited oscillatory peaks that fall within the alpha range, whereas only $4.17 \%$ children but $41.63 \%$ adults had a peak oscillation in the beta band. This suggests that more adults had oscillatory peaks outside of the canonical $8-13 \mathrm{~Hz}$ alpha range. Permutation comparisons showed that for the peak oscillation the centre frequency was significantly higher in adults compared to children (Figure 4B; adults $=13.52 \pm 4.86$, children $=10.25 \pm 3.97, t(46)$ in adults than in children (Figure 4D; adults $=3.63 \pm 3.35$, children $=1.80 \pm 1.13, t(46)=2.53$, $p=0.01 ; \mathrm{BF}=7.07,95 \% \mathrm{Cl}=[0.12,1.2])$

In order to make a valid comparison between parameterised oscillatory components and canonical narrowband analysis, the centre frequency, power, and bandwidth of the highest oscillatory component were also extracted independently for the alpha and beta bands from all individuals (Figure 5A). Figure 5B\&E shows Gaussian curves obtained from the individual oscillatory component values of the FOOOF model for the alpha and beta bands. There is significant individual variability observable for both bands, which is further quantified in Figures 5C, D, F and G. The aperiodic-adjusted beta peak oscillation (but not alpha) was significantly higher in power (Figure 5F; adults $=0.31 \pm 0.11$, children $=0.18 \pm 0.09, t(46)=4.37, p<0.01$; $\mathrm{BF}>100,95 \% \mathrm{Cl}=[0.51,1.77]$ ) and larger in bandwidth (Figure $5 \mathrm{G}$; adults $=4.87 \pm 3.44$, children $=2.39 \pm 2.58, t(46)=2.83, p=0.01 ; \mathrm{BF}=13.01,95 \% \mathrm{Cl}=[0.15,1.29])$ in adults compared to children.

\section{Correlation between Age and Peak Oscillatory Components} in all participants $(r(46)=0.47, \mathrm{BF}=43.08,95 \% \mathrm{Cl}=[0.2,0.65])$, but no such evidence for the individual age groups (adults: $r=0.4, \mathrm{BF}=1.47,95 \% \mathrm{Cl}=[-0.11,0.67]$; children: $r(46)=$ $0.26, \mathrm{BF}=0.51,95 \% \mathrm{Cl}=[-0.16,0.57])$. 
390 In addition, when analysing the periodic components in the beta and alpha bands, Bayes

391 Factors revealed a very strong positive correlation between age and power in the beta band 392 in all participants $(r=0.5, \mathrm{BF}=43.08,95 \% \mathrm{Cl}=[0.2,0.65])$, but substantial evidence for the 393 absence of such a correlation in individual age groups (adults: $r=0.08, \mathrm{BF}=0.27,95 \% \mathrm{Cl}=$

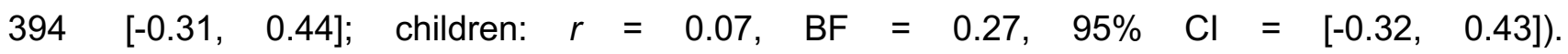
395 In children a strong positive correlation was found between age and centre frequency in the alpha band $(r=0.61, \mathrm{BF}=27.14,95 \% \mathrm{Cl}=[0.24,0.8])$.

\section{Beta-specific Power Increase in the Flattened Parameterised Power Spectra}

398

399

400

401

402

403

404

405

406

407

408

409

410

411

412

413

414

Figure $2 C \& D$ displays the results of conventional spectral analysis applied to the flattened PSD, where the aperiodic signal was removed from the power spectrum, thereby leaving only the oscillatory components. Interestingly, unlike the multiple band-specific power differences found in the raw power spectra (Figure 2B), the permutation analysis only identified significantly increased beta power (Figure $2 \mathrm{D}, t(46)=4.15,<0.01)$ when comparing adults and children. The same frequency ranges (children: 5-13 Hz; adults: $8-13 \mathrm{~Hz}$ ) as for the conventional analyses were applied when estimating peak frequencies in FOOOF. The rightcorner panel in Figure2B shows that there was no significant difference in the peak frequency between adults $(8.78 \pm 3.58 \mathrm{~Hz})$ and children $(9.42 \pm 0.84 \mathrm{~Hz}, t(46)=-0.85, p=0.4)$ in the flattened spectra.

Bayes Factors revealed decisive evidence for larger aperiodic-adjusted power in the beta band (BF $>100,95 \% \mathrm{Cl}=[0.45,1.72])$ in adults compared to children, insufficient evidence for any group difference in the delta and alpha bands (delta: $\mathrm{BF}=0.35,95 \% \mathrm{Cl}=[-0.64,-0.01]$; alpha: $\mathrm{BF}=0.92,95 \% \mathrm{Cl}=[0.02,0.85])$, and strong evidence for the absent group differences in the theta and gamma bands (theta: $\mathrm{BF}=0.17,95 \% \mathrm{Cl}=[-0.46,-0.006]$; gamma: $\mathrm{BF}=0.28,95 \%$ $\mathrm{Cl}=[0.01,0.59])$. Bayes Factors further suggested substantial evidence for absence of group difference for alpha peak frequency $(B F=0.17,95 \% \mathrm{Cl}=[0.01,0.45])$. 
415 Positive Correlation between Aperiodic-adjusted Beta Power and Aperiodic 1/f 416 Component

417 Based on the significant group differences in the aperiodic-adjusted power in the beta band 418 and $1 / f$ signal identified in the parametrised spectra, the presence of correlations between 419 the beta power in the flattened spectra and the parameters of the $1 / f$ signal was assessed. 420 Bayes Factors revealed a very strong correlation between the narrowband aperiodic-adjusted 421 beta power in the flattened spectral and $1 / f$ Slope $(r=0.5, \mathrm{BF}=94.36,95 \% \mathrm{Cl}=[0.24,0.67])$, 422 and $1 / f$ Offset $(r=-0.49, \mathrm{BF}=66.53,95 \% \mathrm{Cl}=[-0.66,-0.22])$, across all participants but not 423 in individual age groups. 

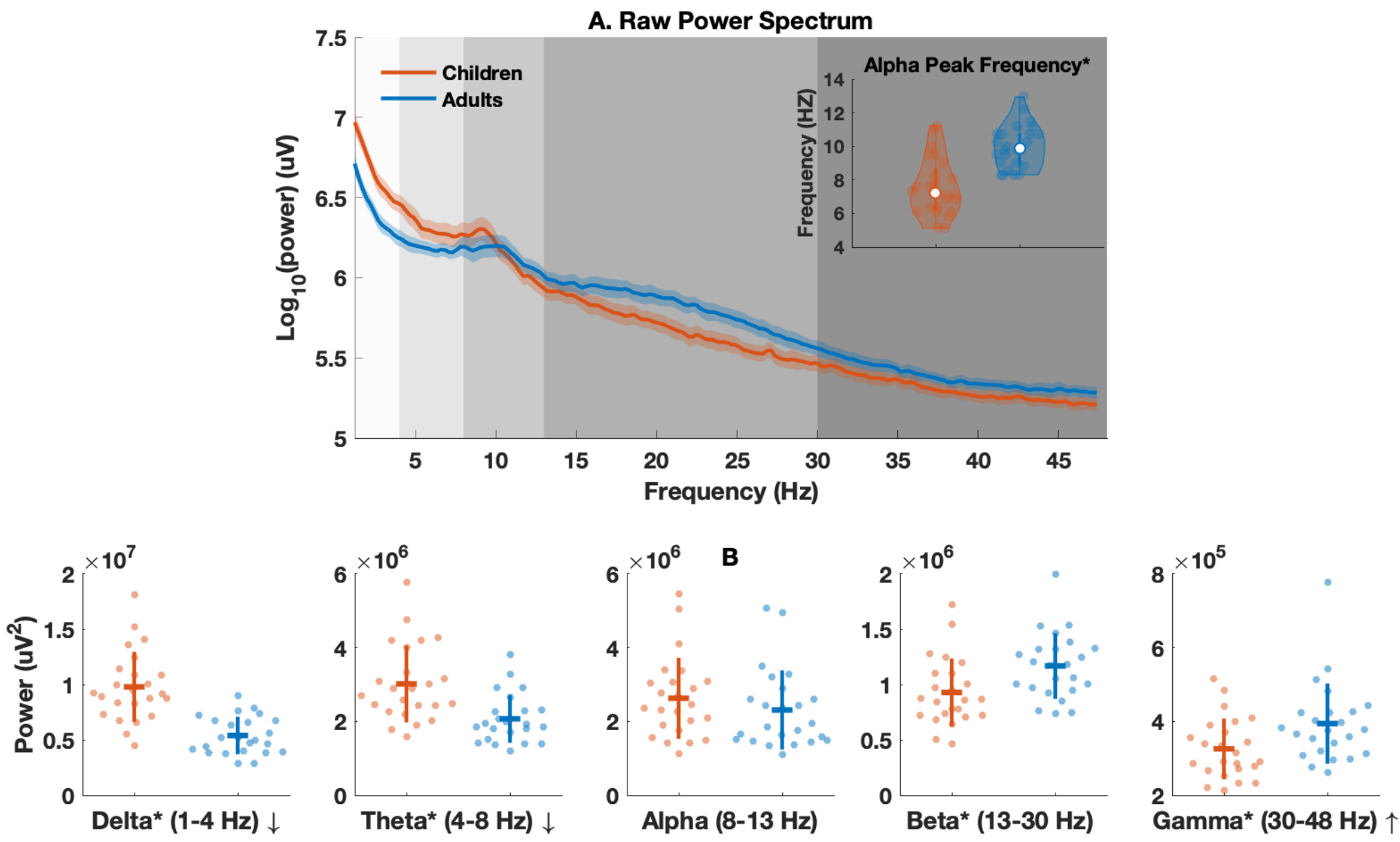
C. Flattened Power Spectrum (1/f Signal Removed)
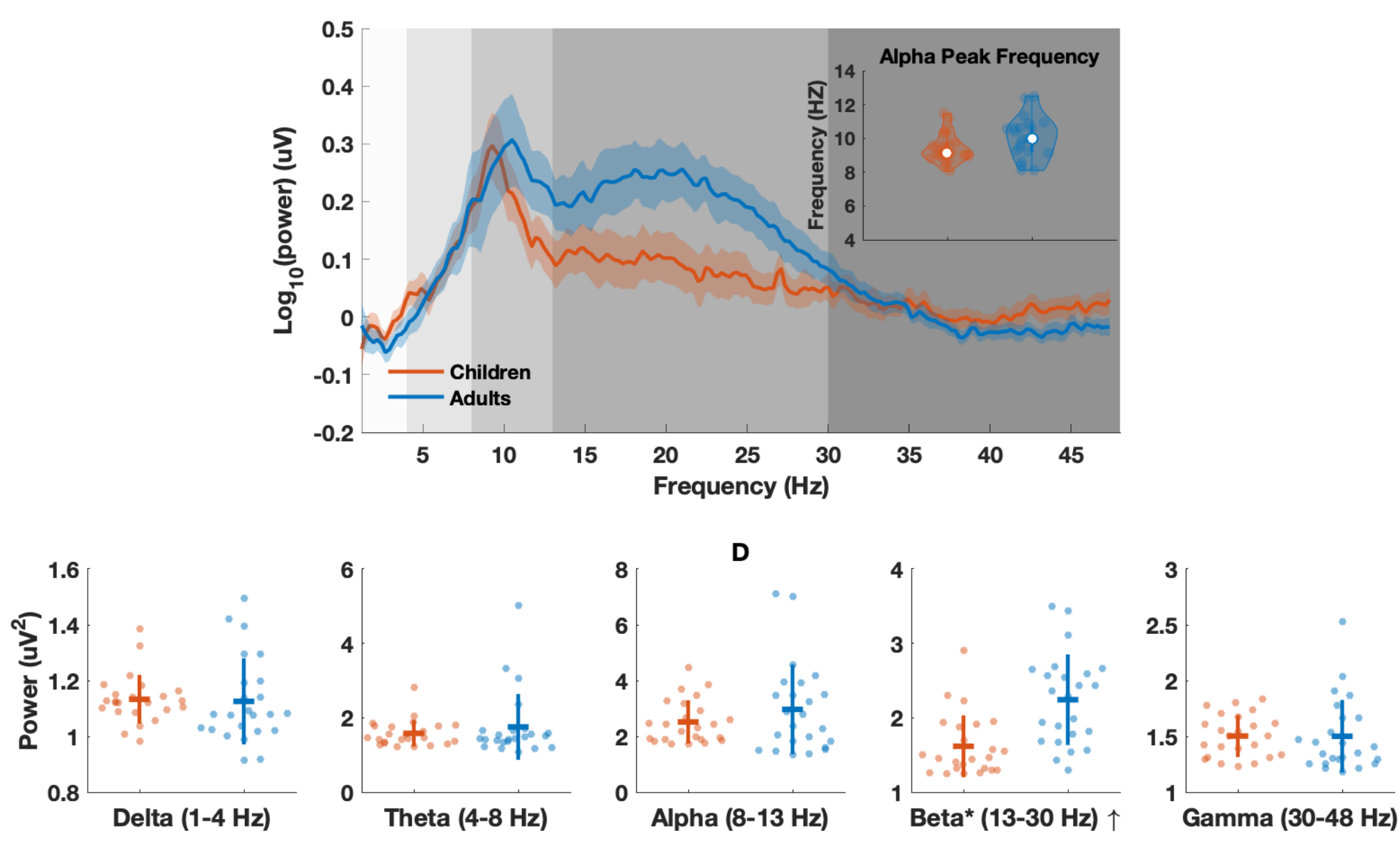
Figure 2. Power Spectral Density for Children (in red, $N=24$ ) and Adults (in blue, $N=24$ ). Grand average of the raw power spectrum (A) and flattened power spectrum (C) for children and adults, with $95 \%$ confidence intervals represented by shaded areas (Gaussian-distribution assumed). Violin plots of the peak frequencies identified in the raw power spectra for children $(5-13 \mathrm{~Hz})$ and adults $(8-13 \mathrm{~Hz})$ are shown in the top-right panels of Figure 2A\&C. The white dots depict group mean values. Power (B) and aperiodic-adjusted power (D) in five a priori defined canonical frequency bands as colour coded regions with grey gradients. Each dot represents a child participant in read or an adult participant in blue. Horizontal lines indicate group mean and vertical lines standard deviations. * indicates group difference reaches statistical significance; $\uparrow$ and $\downarrow$ indicate significant increase and significant decrease in power, respectively. 
A. Reconstructed Aperiodic Power Spectrum

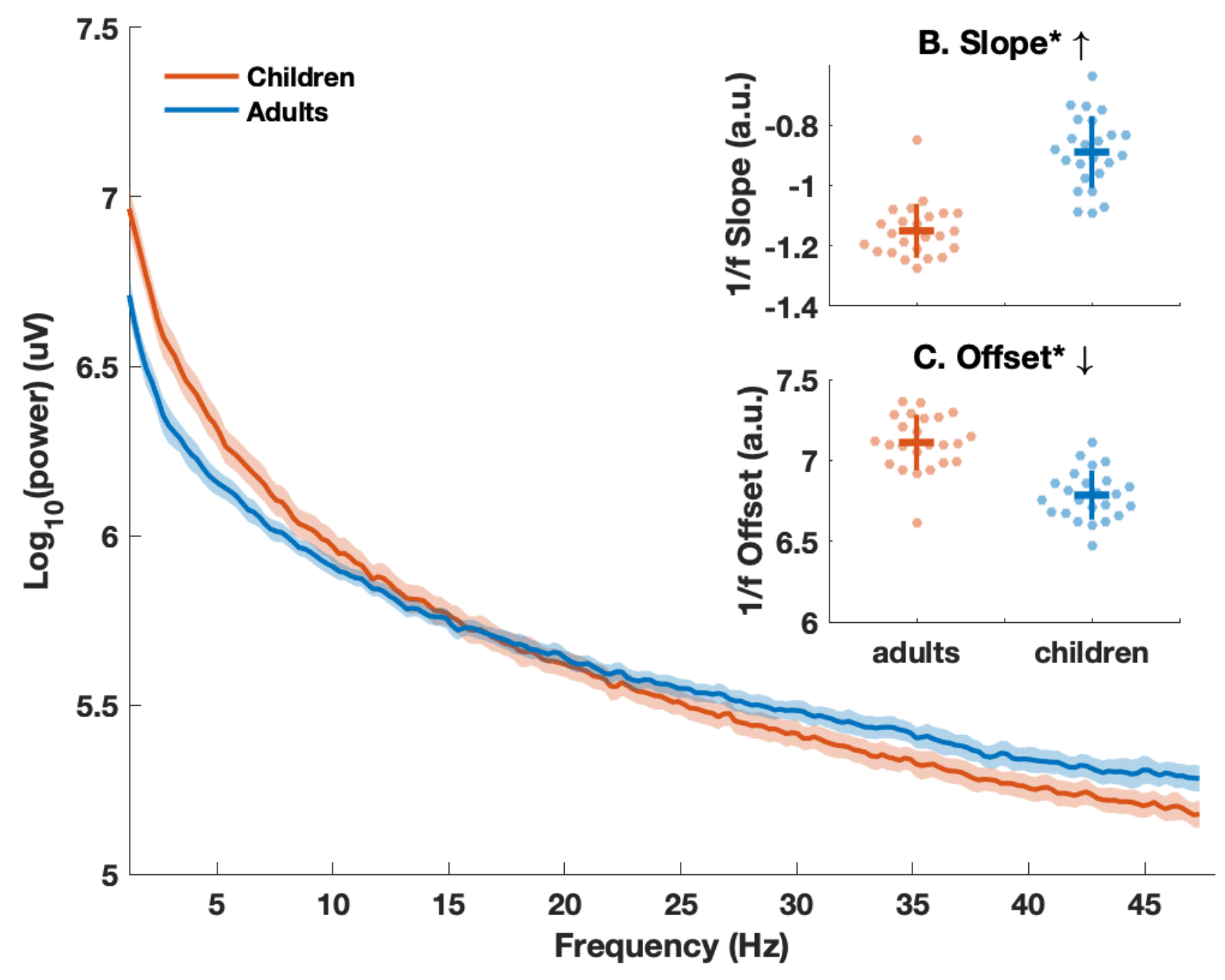


435 Figure 3. Non-oscillatory Power Spectrum (i.e., oscillatory components have been removed) for $\mathrm{Children} \mathrm{(in} \mathrm{red,} \mathrm{N}=24$ ) and 436 Adults (in blue, $\mathbf{N}=24$ ). A: Grand average of the log-transformed power spectrum of the aperiodic signal for children and 437 adults, with $95 \%$ confidence intervals represented by shaded areas (Gaussian-distribution assumed). B\&C: Slope and Offset 438 of the aperiodic power spectrum for both groups. Horizontal lines indicate group mean and vertical lines standard 439 deviations. * indicates group difference reaches statistical significance; $\uparrow$ and $\downarrow$ indicate significant increase and significant 440 decrease in power, respectively. 

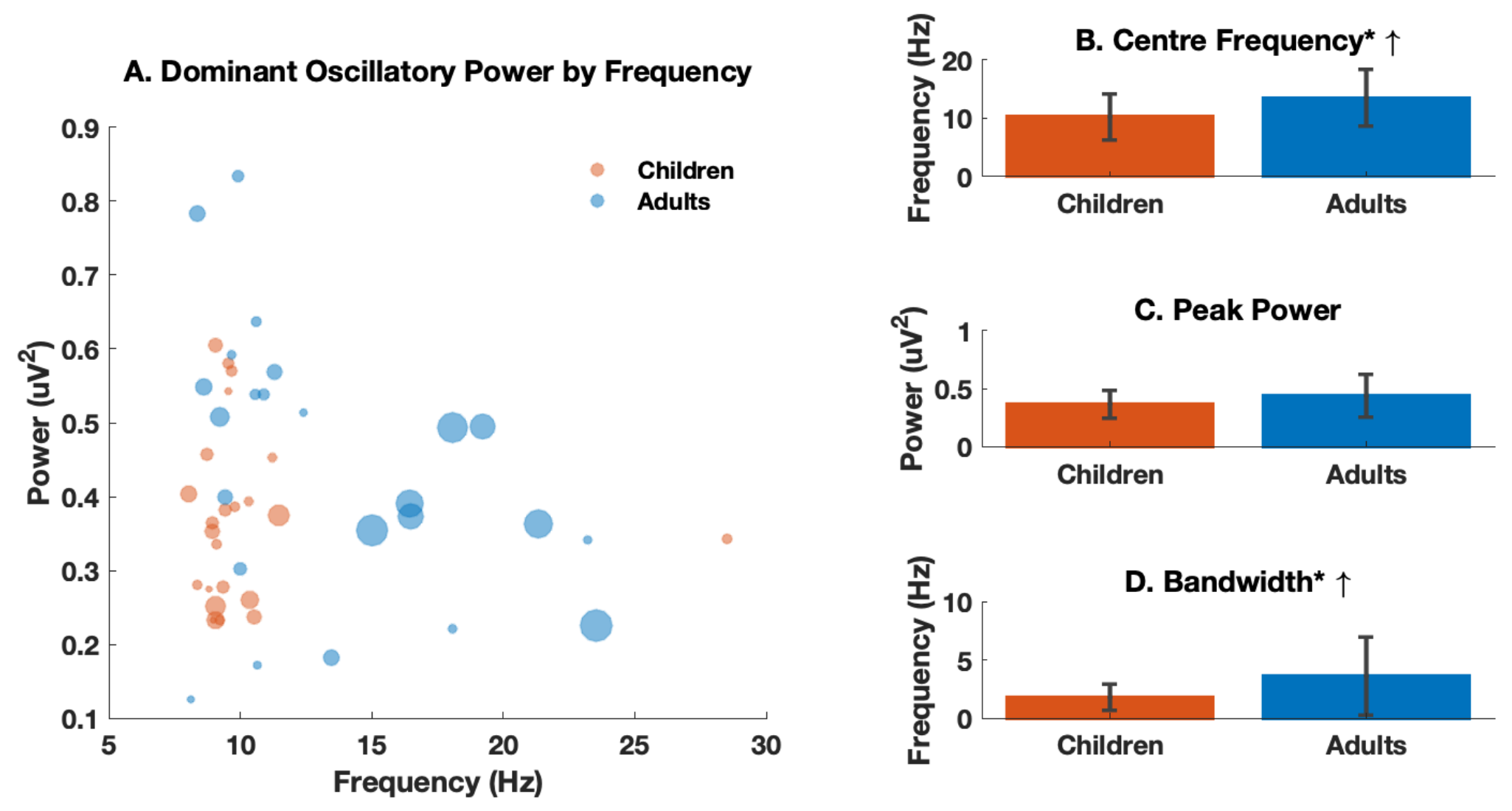
442 Figure 4. Dominant Oscillations in the Parameterised Power Spectrum for Children (in red, $\mathbf{N}=\mathbf{2 4}$ ) and Adults (in blue, $\mathbf{N}=$ 443 24). A: The power of the dominant oscillation (i.e., oscillation with the maximum power across the broadband power 444 spectrum) by frequency for each individual, with the size of the circle representing the bandwidth of the dominant oscillation. 445 B-D: Statistical comparisons of the centre frequency, power and the bandwidth of the dominant oscillation. Error bars 446 represent standard error of the group means. * indicates group difference reaches statistical significance; $\uparrow$ and $\downarrow$ indicate 447 significant increase and significant decrease in power, respectively. 


\section{A. Parameterised Alpha and Beta Oscillations}

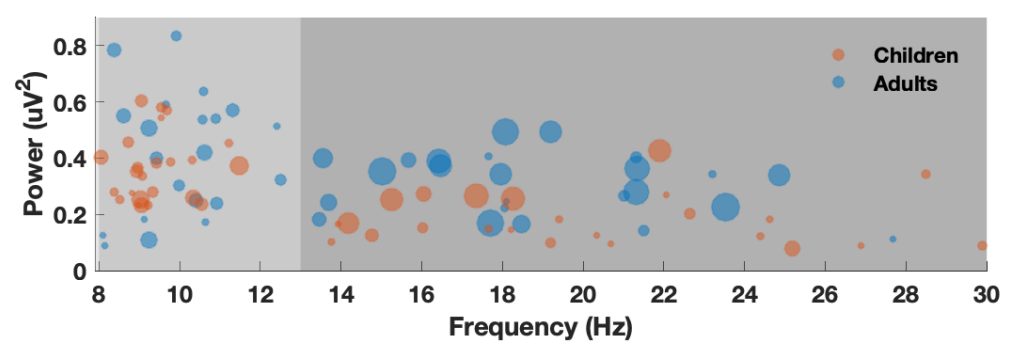

B. Alpha:(8 - 13 Hz)
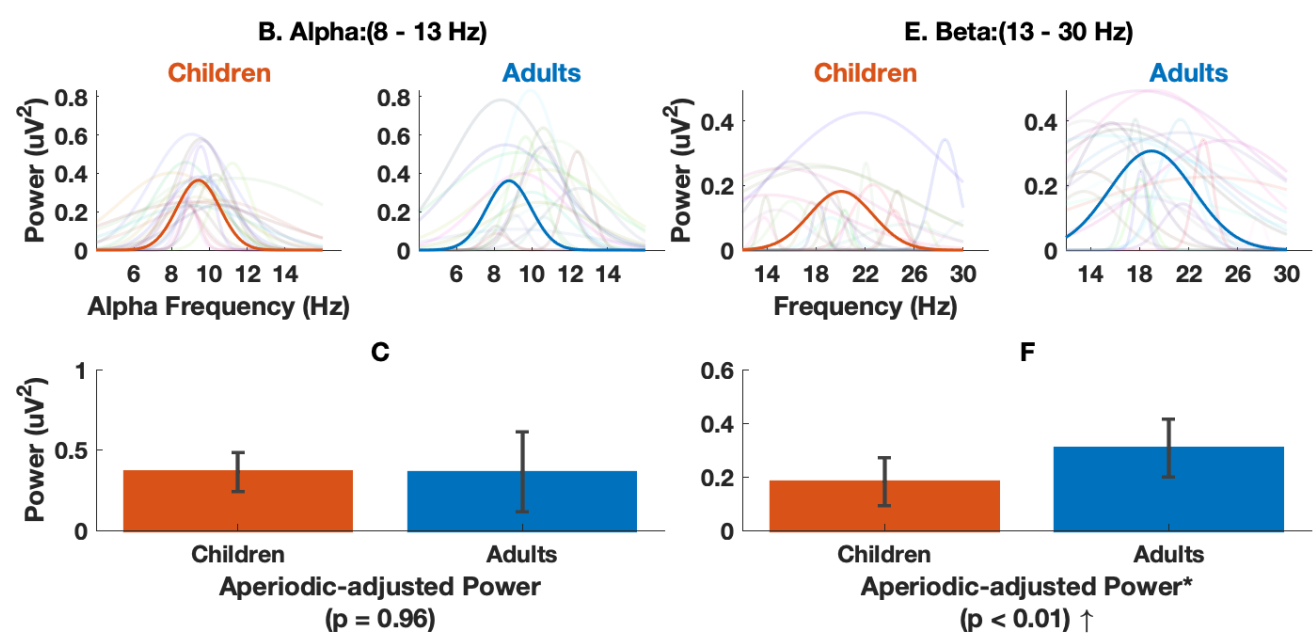

$(p<0.01) \uparrow$
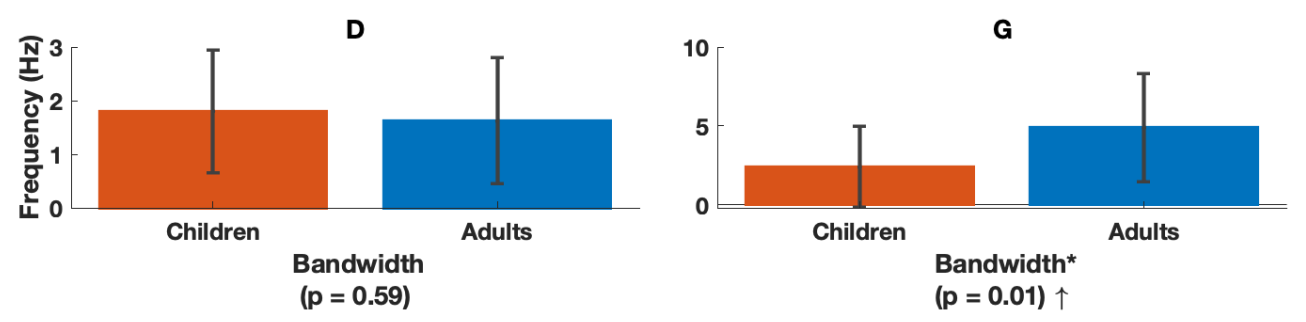

449 Figure 5. Alpha and Beta Band Parameterisation of the Power Spectrum for 450 Children (in red, $\mathbf{N}=24$ ) and Adults (in blue, $\mathbf{N}=24$ ). Two-band models of the power spectrum (A) - alpha (red, 8-13 Hz) and beta (blue, 13-30 Hz). B\&E show the maximum oscillatory power and the frequency at which this occurs for each individual. Statistical comparisons of the power (C\&F) and the bandwidth (D\&G)

454 of the oscillations in the alpha and beta bands. Error bars represent standard 455 error of the group means. frequency, power and the bandwidth of the peak oscillation components. Error bars represent standard error of the group means. 
458 significant increase in power. 


\section{DISCUSSION}

460

461

462

463

464

465

466

467

468

469

470

471

472

473

474

475

476

477

478

479

480

481

482

483

We investigated developmental changes in the aperiodic $1 / f$ and the oscillatory components of MEG brain signals. The findings of our conventional analyses of narrowband power and peak frequency were remarkably similar to previous studies (Marcuse et al., 2008; Boersma et al., 2011; Cragg et al., 2011; Miskovic et al., 2015; Gomez et al., 2017; Rodriguez-Martinez et al., 2017). However, these results turned out to be mostly non-significant (except in the beta band) once the aperiodic $1 / f$ signal and the oscillations were disentangled, and once the narrowband power was assessed in the flattened power spectrum (that is after the $1 / f$ component had been removed as shown in Figure 2). In these non-conventional analyses, we observed distinct (complementary) developmental profiles for the $1 / f$ signal and oscillatory power, with compelling evidence of flatter $1 / f$ signals and increased beta oscillations in the adults, as compared to the children. Moreover, the strong correlation between the $1 / f$ signal and beta oscillatory power suggested a co-maturation of the two neural phenomena during child development.

\section{Conventional versus Parameterised Power Spectral Analyses}

Previous developmental studies have shown power decreases in lower frequency bands (Puligheddu et al., 2005; Gomez et al., 2013; Schafer et al., 2014), and increases in alpha peak frequency throughout childhood (Marcuse et al., 2008; Boersma et al., 2011; Cragg et al., 2011; Smit, Boomsma, et al., 2012; Miskovic et al., 2015; Gomez et al., 2017; RodriguezMartinez et al., 2017). By applying conventional methods, we were able to replicate these findings. However, none of these findings remained significant once careful adjudication between aperiodic $1 / f$ and oscillatory components was carried out using power spectra parameterisation (Haller et al., 2018).

Interestingly, the peak frequency in the flattened spectra was found to correlate positively with age in the child participants only, but the peak frequency showed no differences between age 
groups (Figure 2B), and neither did the aperiodic-adjusted power or frequency bandwidth in 485 the alpha band (Figure 5C\&D). These findings contrast with significant age differences found 486 in the alpha band when not accounting for the aperiodic $1 / f$ signal, and suggest that the 487 magnitude of these changes could be heavily conflated by the aperiodic $1 / f$ signal in the raw 488 power spectrum. This is further supported by the significant correlation that was identified 489 between the $1 / f$ signal (both offset and slope) and age, only in the child participants. These 490 findings invite the conclusion that developmentally-related power decreases in lower frequency bands and peak frequency increases that have been revealed by standard methods are at least partially driven by the flattening of the $1 / f$ component in the broadband spectrum 493 (Voytek and Knight, 2015).

\section{$494 \quad$ Flatter Slope Indicates Increase in Neuronal Noise}

495 The slope of the $1 / f$ signal was found to be flatter, or less negative, in adults than in children. 496 According to the "Wiener-Khinchin theorem", the power spectrum is equivalent to the Fourier 497 transform of the autocovariance function (He, 2014). Therefore, a flatter slope in the frequency 498 domain indicates a shorter/weaker autocorrelation in the time domain. Interestingly, the reduction of the autocorrelation in human brain activity has been found to correlate with the increasing demands for more efficient online information processing during cognitive load in working memory tasks (He, 2014; Voytek et al., 2015). Indeed, a reduced temporal integration span in brain activity would be expected to reflect the need for enhanced integration of information during brain development.

504 There is also evidence that neuronal spiking statistics are relevant to the $1 / f$ slope (Voytek and Knight, 2015; Gao, 2016), in that the slope of the aggregated local field potential becomes flatter when a large number of spikes occur asynchronously (Usher et al., 1995; Pozzorini et al., 2013; Voytek and Knight, 2015). This decoupling of neuronal population spiking from an oscillatory regime, which has been broadly defined as "noise", can be driven by increases in the ratio between local excitation/inhibition (Cremer and Zeef, 1987; McIntosh et al., 2010; 
510 Hong and Rebec, 2012; He, 2014; Voytek et al., 2015). In fact, the inhibitory regulation of the

511 synchronisation between pyramidal neurons from the GABAergic neurons has been found to

512 undergo prolonged changes into adolescence (Hashimoto et al., 2009).

513 The increase of neuronal noise during brain development has also been reported in studies

514 adopting a simplistic method that uses the variability of brain signals as a proxy for neuronal

515 noise. Such efforts have consistently identified increasing variability of both spontaneous and

516 evoked brain activity to correlate with more stable and accurate behaviour during development

517 (Mclntosh et al., 2010; Fransson et al., 2013). Of direct relevance to our findings, a cross-

518 sectional EEG study comparing children aged 8-15 years with young adults reported that the

519 variability of evoked face responses increased with age. This variability turned out to be

520 positively correlated with intrasubject response accuracy but negatively correlated with

521 reaction-time variability (Mclntosh et al., 2008). This increase in neuronal noise in the

522 developing brain appeared to be more global, as compared to a more local noise increase

523 with aging (Mclntosh et al., 2010). Speculatively, neuronal noise during childhood shapes the

524 brain from a deterministic system into one that is more stochastic and adaptive to an uncertain

525 environment (Knill and Pouget, 2004; Stein et al., 2005). Brain noise in the aging population

526 continues to increase, but the pattern of changes can be more focal and is often in parallel

527 with diminished neuroplasticity (Li et al., 2006; Garrett et al., 2010). Such speculation

528 regarding the network level gains support from our recent analysis of brain network topology

529 in the same dataset, where pervasive decreases in connectedness were revealed (i.e., nodal

530 centrality) in most cortical regions, with increasing global network segregation (He et al., 2019).

531 The same approach, however, has also identified quite focal changes in hub regions as the

532 driving force of large-scale network abnormalities in various neurological diseases (Stam et

533 al., 2009; Crossley et al., 2014; DeSalvo et al., 2014; Tewarie et al., 2014; Yu et al., 2017). 


\section{Smaller Offset Indicates Reduction in Broadband Power}

535 Accumulating evidence suggests that the offset of the $1 / f$ signal reflects the broadband power, 536 which in turn is associated with the aggregated spiking activity of the underlying neuronal 537 populations (Manning et al., 2009; Miller et al., 2009; Miller et al., 2014). We found a significant 538 reduction in the offset of the $1 / f$ signal for adults as compared to children, which could indicate 539 a reduction in broadband power. Broadband power reductions have been reported consistently in developmental MEG/EEG studies (Miskovic et al., 2015; Gomez et al., 2017;

541 Rodriguez-Martinez et al., 2017). This finding also corroborates a previous report on the 542 developmental parallelism between the reduction in spectral power and cortical thickness 543 (Whitford et al., 2007). It is understood that brain development begins with neuronal 544 proliferation and synaptogenesis, followed by synaptic pruning during which synapses are 545 selectively eliminated (Marsh et al., 2008). Computational work has shown that MEG signals 546 represent spikes and the envelope of membrane de-/hyper-polarisation, predominantly from pyramidal neurons (Murakami and Okada, 2006). Therefore, one possible mechanism

548 underlying the observed offset decreases is "regressive" cortical organisation due to the loss 549 of grey matter (Giedd et al., 1999; Sowell et al., 2003).

550 We note here that our interpretation of the reduced $1 / f$ offset needs to be taken with caution.

551 This is because slope and offset of the $1 / f$ signal are highly correlated (Haller et al., 2018), 552 and thus any change in slope would be accompanied by a change in the offset, regardless of 553 the offset shifts caused by broadband power reductions. Future studies are needed to clarify 554 the extent to which the observed offset differences between age groups were caused over 555 and above those expected from slope shifts.

\section{Co-maturing Beta Oscillations during Childhood}

557 Analyses of oscillatory power revealed larger beta power in adults, as compared to children, 558 and the difference remained significant once the $1 / f$ signal was removed from the broadband 
power spectrum (Figures 6). The automatic parameterisation algorithm also revealed age-

560 group differences in both peak oscillatory power and bandwidth in the beta band (Figures 5).

561 Our findings add to previous developmental evidence for an increase in beta power using resting-state MEG/EEG (Puligheddu et al., 2005; Gomez et al., 2013; Schafer et al., 2014; Khan et al., 2018), and concurrent EEG-fMRI (Luchinger et al., 2011). These findings are also in accord with our recent longitudinal MEG study of motor development in children, which demonstrated linear increases in amplitude and mean frequency of beta (but not gamma) in movement (Johnson et al., 2019). Indeed, the beta band has been found to be heavily engaged in a wide range of processes such as cognitive control (Buschman and Miller, 2014), which develop well into adolescence (Luna et al., 2015) and changes with aging (Xifra-Porxas et al., 2019).

It is also worth noting that, in contrast to functional resonance imaging (fMRI), the MEG signal has a more direct relationship with neuronal activity and, compared to EEG signal, is less contaminated by age-related changes of structures external to the brain, such as the decreasing electrical conductivity of the human skull with age (Hamalainen et al., 1993; Hoekema et al., 2003; Baillet, 2017). Therefore, the increased absolute beta power detected by MEG reinforces the neuronal origin of the maturational power change reported by EEG and fMRI (Gasser et al., 1988; Marcuse et al., 2008; Boersma et al., 2011; Cragg et al., 2011; Smit, Boomsma, et al., 2012; Bathelt et al., 2013; Fransson et al., 2013; Miskovic et al., 2015;

578 Gomez et al., 2017; Rodriguez-Martinez et al., 2017; Vandenbosch et al., 2019). In future work, 579 we plan to determine if the current findings can be replicated in larger (longitudinal) data sets, and we will attempt to establish a more fine-tuned cortical representation of the $1 / f$ and oscillatory signals from power spectra parameterisation. We encourage others to collect or reanalyse their resting-state data using the techniques we have employed (e.g., https://github.com/fooof-tools/fooof/), as well as other open source tools, to help establish norms of neuronal oscillations and aperiodic signals that are characteristic of healthy brain development. 


\section{Limitations}

587 There are some limitations to the present study. First, although our study provides valuable 588 insights into age-group differences in key measures of neuronal activity, the cross-sectional 589 nature of the study did not permit us to observe longitudinal changes at an individual level. A 590 large longitudinal sample with balanced gender would be expected to replicate the current 591 findings. Second, we assessed age-related differences based on 15 clean trials per participant. 592 This was done to counteract differences in the number of clean trials between the adult and 593 child participants. Data from the younger participants were prone to a greater number of 594 artefacts, such as in-scanner motion. Future study should make use of a more efficient data 595 acquisition approach (e.g., using calming video clips (Vanderwal et al., 2015)) to obtain larger 596 data samples with improved quality in young children (Rapaport et al., 2019).

\section{Conclusions}

598 By carefully modelling the power spectra of source-space electrophysiological data in adults 599 and in children aged 4 to 12 years, the present study demonstrated strong evidence of correlated increases in the $1 / f$ signal and beta power during child brain development. Our findings suggest that the reported power decreases in low-frequencies and the reported power increases in high-frequencies (other than the beta band) in many previous studies could have

603 been caused by a flattening of the $1 / f$ signal, instead of an authentic power change in the 604 oscillations. In addition, our findings provide empirical support for the theory that neuronal 605 noise increases with normal brain maturation (Mclntosh et al., 2010). This change may shape 606 the brain into a more stochastic system with balanced neuronal excitation/inhibition, greater 607 complexity, and greater capacity for information processing (Knill and Pouget, 2004; Stein et 608 al., 2005). Overall, the findings of the present study suggest that co-increasing beta oscillations and aperiodic brain signals accompany brain maturation during childhood. 


\section{ACKNOWLEDGEMENTS}

611 We thank all participants for their participation. We also thank Douglas Cheyne and Cecilia

612 Jobst for their assistance in the preliminary data analysis, and Craig Richardson for his

613 technical support in establishing inter-institutional collaborative data analysis platform. Finally,

614 we acknowledge the collaboration of Kanazawa Institute of Technology in establishing the

615 KIT-Macquarie MEG laboratory. This work was supported by the Australian Research Council

616 (ARC) Centre of Excellence in Cognition and its Disorders (grant number CE110001021,

617 http://www.ccd.edu.au), and the ARC Discovery Project (DP170103148). Wei He was

618 supported by the Macquarie University Research Fellowship (\#9201501199). Paul F. Sowman

619 was supported by the ARC Discovery Early Career Researcher Award (DE130100868) and

620 the Australian National Health and Medical Research Council (\#1003760). 


\section{1}

622

623

624

625

626

627

628

629

630

631

632

633

634

635

636

637

638

639

640

641

642

\section{REFERENCES}

Amzica F, Steriade M (1998) Electrophysiological correlates of sleep delta waves. Electroencephalogr Clin Neurophysiol, 107:69-83.

Baillet S (2017) Magnetoencephalography for brain electrophysiology and imaging. Nat Neurosci, 20:327-339.

Bak P, Tang C, Wiesenfeld K (1987) Self-organized criticality: An explanation of the 1/f noise. Phys Rev Lett, 59:381-384.

Bathelt J, O'Reilly H, Clayden JD, Cross JH, de Haan M (2013) Functional brain network organisation of children between 2 and 5 years derived from reconstructed activity of cortical sources of high-density eeg recordings. Neuroimage, 82:595-604.

Benasich AA, Gou Z, Choudhury N, Harris KD (2008) Early cognitive and language skills are linked to resting frontal gamma power across the first 3 years. Behav Brain Res, 195:215-222.

Berchicci M, Zhang T, Romero L, Peters A, Annett R, Teuscher U, Bertollo M, Okada Y, Stephen J, Comani S (2011) Development of mu rhythm in infants and preschool children. Dev Neurosci, 33:130-143.

Boersma M, Smit DJ, de Bie HM, Van Baal GC, Boomsma DI, de Geus EJ, Delemarre-van de Waal HA, Stam CJ (2011) Network analysis of resting state eeg in the developing young brain: Structure comes with maturation. Hum Brain Mapp, 32:413-425.

Boord PR, Rennie CJ, Williams LM (2007) Integrating "brain" and "body" measures: Correlations between eeg and metabolic changes over the human lifespan. $J$ Integr Neurosci, 6:205-218. 
643 Brookes MJ, Vrba J, Robinson SE, Stevenson CM, Peters AM, Barnes GR, Hillebrand A, 644 Morris PG (2008) Optimising experimental design for meg beamformer imaging.

645 Neuroimage, 39:1788-1802.

646 Burianova H, Marstaller L, Sowman P, Tesan G, Rich AN, Williams M, Savage G, Johnson

647

648 BW (2013) Multimodal functional imaging of motor imagery using a novel paradigm.

Buschman TJ, Miller EK (2014) Goal-direction and top-down control. Philos Trans R Soc Lond

650 B Biol Sci, 369.

651

Campbell IG, Feinberg I (2009) Longitudinal trajectories of non-rapid eye movement delta and

652

653 theta eeg as indicators of adolescent brain maturation. Proc Natl Acad Sci U S A, 106:5177-5180.

654

Cheyne D, Bostan AC, Gaetz W, Pang EW (2007) Event-related beamforming: A robust 655 method for presurgical functional mapping using meg. Clin Neurophysiol, 118:1691-

656 1704.

657

Cheyne D, Ferrari P (2013) Meg studies of motor cortex gamma oscillations: Evidence for a 658 gamma "fingerprint" in the brain? Front Hum Neurosci, 7:575.

659

Clayton MS, Yeung N, Cohen Kadosh R (2018) The many characters of visual alpha 660 oscillations. Eur J Neurosci, 48:2498-2508.

661

Cragg L, Kovacevic N, Mclntosh AR, Poulsen C, Martinu K, Leonard G, Paus T (2011) 662 Maturation of eeg power spectra in early adolescence: A longitudinal study. Dev Sci, 663 14:935-943. 
665 Crossley NA, Mechelli A, Scott J, Carletti F, Fox PT, McGuire P, Bullmore ET (2014) The hubs of the human connectome are generally implicated in the anatomy of brain disorders. Brain, 137:2382-2395.

668

DeSalvo MN, Douw L, Tanaka N, Reinsberger C, Stufflebeam SM (2014) Altered structural 669 connectome in temporal lobe epilepsy. Radiology, 270:842-848.

670

Dienes Z (2011) Bayesian versus orthodox statistics: Which side are you on? Perspect 671 Psychol Sci, 6:274-290.

672 Dienes Z (2014) Using bayes to get the most out of non-significant results. Front Psychol, 5.

673 Etchell AC, Ryan M, Martin E, Johnson BW, Sowman PF (2016) Abnormal time course of low 674

675 beta modulation in non-fluent preschool children: A magnetoencephalographic study of rhythm tracking. Neuroimage, 125:953-963.

676

Fransson P, Metsaranta M, Blennow M, Aden U, Lagercrantz H, Vanhatalo S (2013) Early 677 development of spatial patterns of power-law frequency scaling in fmri resting-state

678 and eeg data in the newborn brain. Cereb Cortex, 23:638-646.

679

Gaetz W, MacDonald M, Cheyne D, Snead OC (2010) Neuromagnetic imaging of movement680 681 related cortical oscillations in children and adults: Age predicts post-movement beta rebound. Neuroimage, 51:792-807.

682

Gao R (2016) Interpreting the electrophysiological power spectrum. J Neurophysiol, 115:628-

683 630.

684 Gao R, Peterson EJ, Voytek B (2017) Inferring synaptic excitation/inhibition balance from field 685 potentials. Neuroimage, 158:70-78. variability is more than just noise. Journal of Neuroscience, 30:4914-4921. 
688 Gasser T, Verleger R, Bacher P, Sroka L (1988) Development of the eeg of school-age children and adolescents. I. Analysis of band power. Electroencephalogr Clin Neurophysiol, 69:91-99.

691

692

693

694

695

696

697

698

699

700

701

702

703

704

705

706

707

708

709

710

Giedd JN, Blumenthal J, Jeffries NO, Castellanos FX, Liu H, Zijdenbos A, Paus T, Evans AC, Rapoport JL (1999) Brain development during childhood and adolescence: A longitudinal mri study. Nat Neurosci, 2:861-863.

Gomez C, Perez-Macias JM, Poza J, Fernandez A, Hornero R (2013) Spectral changes in spontaneous meg activity across the lifespan. J Neural Eng, 10:066006.

Gomez CM, Rodriguez-Martinez El, Fernandez A, Maestu F, Poza J, Gomez C (2017) Absolute power spectral density changes in the magnetoencephalographic activity during the transition from childhood to adulthood. Brain Topogr, 30:87-97.

Haegens S, Cousijn H, Wallis G, Harrison PJ, Nobre AC (2014) Inter- and intra-individual variability in alpha peak frequency. Neuroimage, 92:46-55.

Haller M, Donoghue T, Peterson E, Varma P, Sebastian P, Gao R, Noto T, Knight RT, Shestyuk A, Voytek B (2018) Parameterizing neural power spectra. bioRxiv:299859.

Hamalainen M, Hari R, Ilmoniemi RJ, Knuutila J, Lounasmaa OV (1993) Magnetoencephalography - theory, instrumentation, and applications to noninvasive studies of the working human brain. Rev Mod Phys, 65:413-497.

Hashimoto T, Nguyen QL, Rotaru D, Keenan T, Arion D, Beneyto M, Gonzalez-Burgos G, Lewis DA (2009) Protracted developmental trajectories of gabaa receptor alpha1 and alpha2 subunit expression in primate prefrontal cortex. Biol Psychiatry, 65:1015-1023.

He BJ (2014) Scale-free brain activity: Past, present, and future. Trends Cogn Sci, 18:480487. 
711 He BJ, Zempel JM, Snyder AZ, Raichle ME (2010) The temporal structures and functional significance of scale-free brain activity. Neuron, 66:353-369.

713 He W, Brock J, Johnson BW (2015) Face processing in the brains of pre-school aged children measured with meg. Neuroimage, 106:317-327.

715 He W, Garrido MI, Sowman PF, Brock J, Johnson BW (2015) Development of effective connectivity in the core network for face perception. Hum Brain Mapp, 36:2161-2173.

717 He W, Sowman PF, Brock J, Etchell AC, Stam CJ, Hillebrand A (2019) Increased segregation of functional networks in developing brains. Neurolmage, 200:607-620.

Heinrichs-Graham E, McDermott TJ, Mills MS, Wiesman AI, Wang YP, Stephen JM, Calhoun VD, Wilson TW (2018) The lifespan trajectory of neural oscillatory activity in the motor system. Dev Cogn Neurosci, 30:159-168.

722

Hillebrand A, Barnes GR, Bosboom JL, Berendse HW, Stam CJ (2012) Frequency-dependent functional connectivity within resting-state networks: An atlas-based meg beamformer solution. Neuroimage, 59:3909-3921.

Hillebrand A, Tewarie P, van Dellen E, Yu M, Carbo EW, Douw L, Gouw AA, van Straaten EC, Stam CJ (2016) Direction of information flow in large-scale resting-state networks is frequency-dependent. Proc Natl Acad Sci U S A, 113:3867-3872.

Hoekema R, Wieneke GH, Leijten FS, van Veelen CW, van Rijen PC, Huiskamp GJ, Ansems J, van Huffelen AC (2003) Measurement of the conductivity of skull, temporarily removed during epilepsy surgery. Brain Topogr, 16:29-38.

731 Hong SL, Rebec GV (2012) A new perspective on behavioral inconsistency and neural noise 
734 Irimia A, Erhart MJ, Brown TT (2014) Variability of magnetoencephalographic sensor sensitivity measures as a function of age, brain volume and cortical area. Clin Neurophysiol, 125:1973-1984.

737

Jeffreys $H(1998)$ The theory of probability. Oxford: OUP.

738

Jenkinson N, Brown P (2011) New insights into the relationship between dopamine, beta oscillations and motor function. Trends Neurosci, 34:611-618.

Jensen O, Mazaheri A (2010) Shaping functional architecture by oscillatory alpha activity: Gating by inhibition. Front Hum Neurosci, 4.

Johnson B, Jobst C, Al-Loos R, He W, Cheyne D (2019) Developmental changes in movement related brain activity in early childhood. . bioRxiv, :531905.

Johnson BW, Crain S, Thornton R, Tesan G, Reid M (2010) Measurement of brain function in pre-school children using a custom sized whole-head meg sensor array. Clin Neurophysiol, 121:340-349.

Kado H, Higuchi M, Shimogawara M, Haruta Y, Adachi Y, Kawai J, Ogata H, Uehara G (1999) Magnetoencephalogram systems developed at kit. IEEE Trans Appl Supercond, 9:4057-4062.

Khan S, Hashmi JA, Mamashli F, Michmizos K, Kitzbichler MG, Bharadwaj H, Bekhti Y, Ganesan S, Garel KA, Whitfield-Gabrieli S, Gollub RL, Kong J, Vaina LM, Rana KD, Stufflebeam SM, Hamalainen MS, Kenet T (2018) Maturation trajectories of cortical resting-state networks depend on the mediating frequency band. Neuroimage, 174:5768.

Klimesch W (1999) Eeg alpha and theta oscillations reflect cognitive and memory performance: A review and analysis. Brain Res Rev, 29:169-195. 
757 Knill DC, Pouget A (2004) The bayesian brain: The role of uncertainty in neural coding and computation. Trends in Neurosci, 27:712-719.

759

Li SC, Brehmer Y, Shing YL, Werkle-Bergner M, Lindenberger U (2006) Neuromodulation of 760 associative and organizational plasticity across the life span: Empirical evidence and neurocomputational modeling. Neurosci Biobehav Rev, 30:775-790.

762

Luchinger R, Michels L, Martin E, Brandeis D (2011) Eeg-bold correlations during 763 (post-)adolescent brain maturation. Neuroimage, 56:1493-1505.

764

765

766

767

768

769

770

771

772

773

774

775

776

777

778

779

Luna B, Marek S, Larsen B, Tervo-Clemmens B, Chahal R (2015) An integrative model of the maturation of cognitive control. Annu Rev Neurosci, 38:151-170.

Mackay WA (1997) Synchronized neuronal oscillations and their role in motor processes. Trends Cogn Sci, 1:176-183.

Manning JR, Jacobs J, Fried I, Kahana MJ (2009) Broadband shifts in local field potential power spectra are correlated with single-neuron spiking in humans. J Neurosci, 29:13613-13620.

Marcuse LV, Schneider M, Mortati KA, Donnelly KM, Arnedo V, Grant AC (2008) Quantitative analysis of the eeg posterior-dominant rhythm in healthy adolescents. Clin Neurophysiol, 119:1778-1781.

Markand ON (1990) Alpha rhythms. J Clin Neurophysiol, 7:163-189.

Marsh R, Gerber AJ, Peterson BS (2008) Neuroimaging studies of normal brain development and their relevance for understanding childhood neuropsychiatric disorders. $J A m$ Acad Child Adolesc Psychiatry, 47:1233-1251.

Marstaller L, Burianova H, Sowman PF (2014) High gamma oscillations in medial temporal lobe during overt production of speech and gestures. PLoS One, 9:e111473. 
780

McIntosh AR, Kovacevic N, Itier RJ (2008) Increased brain signal variability accompanies lower behavioral variability in development. PLoS Comput Biol, 4.

McIntosh AR, Kovacevic N, Lippe S, Garrett D, Grady C, Jirsa V (2010) The development of a noisy brain. Archives Italiennes De Biologie, 148:323-337.

Mierau A, Felsch M, Hulsdunker T, Mierau J, Bullermann P, Weiss B, Struder HK (2016) The interrelation between sensorimotor abilities, cognitive performance and individual eeg alpha peak frequency in young children. Clin Neurophysiol, 127:270-276.

Miller KJ, Honey CJ, Hermes D, Rao RP, denNijs M, Ojemann JG (2014) Broadband changes in the cortical surface potential track activation of functionally diverse neuronal populations. Neuroimage, 85 Pt 2:711-720.

Miller KJ, Sorensen LB, Ojemann JG, den Nijs M (2009) Power-law scaling in the brain surface electric potential. PLoS Comput Biol, 5:e1000609.

Miskovic V, Ma X, Chou CA, Fan M, Owens M, Sayama H, Gibb BE (2015) Developmental changes in spontaneous electrocortical activity and network organization from early to late childhood. Neuroimage, 118:237-247.

Murakami S, Okada Y (2006) Contributions of principal neocortical neurons to magnetoencephalography and electroencephalography signals. J Physiol, 575:925936.

Podvalny E, Noy N, Harel M, Bickel S, Chechik G, Schroeder CE, Mehta AD, Tsodyks M, Malach R (2015) A unifying principle underlying the extracellular field potential spectral responses in the human cortex. J Neurophysiol, 114:505-519.

Pozzorini C, Naud R, Mensi S, Gerstner W (2013) Temporal whitening by power-law adaptation in neocortical neurons. Nat Neurosci, 16:942-U216. 
803 Puligheddu M, de Munck JC, Stam CJ, Verbunt J, de Jongh A, van Dijk BW, Marrosu F (2005)

804

805

Age distribution of meg spontaneous theta activity in healthy subjects. Brain Topogr,

806

Quintana DS, Williams DR (2018) Bayesian alternatives for common null-hypothesis

807 significance tests in psychiatry: A non-technical guide using jasp. BMC Psychiatry, 18.

808

Raftery AE (1995) Bayesian model selection in social research. Sociol Methodol, 25:111-163.

809

Rapaport H, Seymour RA, Sowman PF, Benikos N, Stylianou E, Johnson B, Crain S, He W

810

(2019) Studying brain function in children using magnetoencephalography. JOVE, 146.

Ritter P, Moosmann M, Villringer A (2009) Rolandic alpha and beta eeg rhythms' strengths are inversely related to fmri-bold signal in primary somatosensory and motor cortex. Hum Brain Mapp, 30:1168-1187.

814 Robinson SE (1999) Functional neuroimaging by synthetic aperture magnetometry (sam). Recent Adv Biomagnetism:302-305.

816 Rodriguez-Martinez El, Ruiz-Martinez FJ, Barriga Paulino Cl, Gomez CM (2017) Frequency shift in topography of spontaneous brain rhythms from childhood to adulthood. Cogn Neurodyn, 11:23-33.

819 Samaha J, Postle BR (2015) The speed of alpha-band oscillations predicts the temporal resolution of visual perception. Curr Biol, 25:2985-2990.

821 Schafer CB, Morgan BR, Ye AX, Taylor MJ, Doesburg SM (2014) Oscillations, networks, and 5261. 
824 Smit DJ, Boomsma DI, Schnack HG, Hulshoff Pol HE, de Geus EJ (2012) Individual differences in eeg spectral power reflect genetic variance in gray and white matter volumes. Twin Res Hum Genet, 15:384-392.

827

828

829

830

831

832

833

834

835

836

837

838

839

840

841

842

843

Smit DJ, Boersma M, Schnack HG, Micheloyannis S, Boomsma DI, Pol HEH, Stam CJ, de Geus EJC (2012) The brain matures with stronger functional connectivity and decreased randomness of its network. Plos One, 7.

Sowell ER, Peterson BS, Thompson PM, Welcome SE, Henkenius AL, Toga AW (2003) Mapping cortical change across the human life span. Nat Neurosci, 6:309-315.

Sowman PF, Dueholm SS, Rasmussen JH, Mrachacz-Kersting N (2014) Induction of plasticity in the human motor cortex by pairing an auditory stimulus with tms. Front Hum Neurosci, 8:398.

Stam CJ, de Haan W, Daffertshofer A, Jones BF, Manshanden I, van Cappellen van Walsum AM, Montez T, Verbunt JP, de Munck JC, van Dijk BW, Berendse HW, Scheltens P (2009) Graph theoretical analysis of magnetoencephalographic functional connectivity in alzheimer's disease. Brain, 132:213-224.

Stein RB, Gossen ER, Jones KE (2005) Neuronal variability: Noise or part of the signal? Nat Rev Neurosci, 6:389-397.

Tewarie P, Hillebrand A, Schoonheim MM, van Dijk BW, Geurts JJG, Barkhof F, Polman CH, Stam CJ (2014) Functional brain network analysis using minimum spanning trees in multiple sclerosis: An meg source-space study. Neurolmage, 88:308-318.

844 Thut G, Miniussi C, Gross J (2012) The functional importance of rhythmic activity in the brain. Curr Biol, 22:R658-663. 
846 Tzourio-Mazoyer N, Landeau B, Papathanassiou D, Crivello F, Etard O, Delcroix N, Mazoyer

847 B, Joliot M (2002) Automated anatomical labeling of activations in spm using a 848 macroscopic anatomical parcellation of the mni mri single-subject brain. Neuroimage,

849 15:273-289.

850 Usher M, Stemmler M, Olami Z (1995) Dynamic pattern formation leads to $1 / \mathrm{f}$ noise in neural populations. Phys Rev Lett, 74:326-329.

852

Valdés P, Biscay R, Galán L, Bosch J, Szava S, Virués T (1990) High resolution spectral eeg 853 norms for topography. Brain Topogr, 3:281-282.

854

Vandenbosch MMLJZ, van t'Ent D, Boomsma DI, Anokhin AP, Smit DJA (2019) Eeg-based

855 age-prediction models as stable and heritable indicators of brain maturational level in children and adolescents. Hum Brain Mapp, 40:1919-1926.

Vanderwal T, Kelly C, Eilbott J, Mayes LC, Castellanos FX (2015) Inscapes: A movie paradigm 858 to improve compliance in functional magnetic resonance imaging. Neuroimage,

859 $122: 222-232$.

860

Voytek B, Knight RT (2015) Dynamic network communication as a unifying neural basis for 861 cognition, development, aging, and disease. Biol Psychiatry, 77:1089-1097.

Voytek B, Kramer MA, Case J, Lepage KQ, Tempesta ZR, Knight RT, Gazzaley A (2015) Age863 related changes in 1/f neural electrophysiological noise. J Neurosci, 35:13257-13265. R, Gazzaley A (2017) Preparatory encoding of the fine scale of human spatial attention. J Cogn Neurosci, 29:1302-1310. Bull Rev, 14:779-804. 
869 Wang C, Ulbert I, Schomer DL, Marinkovic K, Halgren E (2005) Responses of human anterior cingulate cortex microdomains to error detection, conflict monitoring, stimulusresponse mapping, familiarity, and orienting. J Neurosci, 25:604-613.

872 Welch P (1967) The use of fast fourier transform for the estimation of power spectra: A method

873 based on time averaging over short, modified periodograms. IEEE Trans Acoust,

874 15:70-73.

Whitford TJ, Rennie CJ, Grieve SM, Clark CR, Gordon E, Williams LM (2007) Brain maturation

876 in adolescence: Concurrent changes in neuroanatomy and neurophysiology. Hum

877 Brain Mapp, 28:228-237.

Xifra-Porxas A, Niso G, Lariviere S, Kassinopoulos M, Baillet S, Mitsis GD, Boudrias MH (2019) Older adults exhibit a more pronounced modulation of beta oscillations when performing sustained and dynamic handgrips. Neuroimage, 201:116037.

881 Yu M, Engels MMA, Hillebrand A, van Straaten ECW, Gouw AA, Teunissen C, van der Flier WM, Scheltens P, Stam CJ (2017) Selective impairment of hippocampus and posterior hub areas in alzheimer's disease: An meg-based multiplex network study. Brain, 